\title{
Evaluation of Multi-Component Electrocardiogram Beat Detection Algorithms: Implications of Three Different Noise Artifacts
}

\author{
T Last, CD Nugent, FJ Owens, DD Finlay
}

Faculty of Engineering, University of Ulster, UK

\begin{abstract}
Motion artifacts, caused by changes in the electrodeskin impedance, electromyographic (EMG) interference, caused by muscle contractions, and possible baseline drifts are three of the most common sources of noise present in ECG recordings. The present study investigates the effects of these noise sources on the performance of ECG beat detection algorithms. Four different beat detection methods were used to evaluate the influence of noise sources with varying signal to noise ratios (SNRs). A database consisting of recordings from approximately 100 subjects consisting of approximately 3000 cardiac cycles was used for evaluation. Hence, 1200 records were subsequently tested by the detectors after adding three different noise sources with four different SNRs of $24 d B, 12 d B, 6 d B$ and $-6 d B$ to the original 100 records. The four classifiers achieved beat detection results from $98 \%$ down to $68 \%$ for correctly detected QRS-complexes at SNRs between $24 \mathrm{~dB}$ and $6 \mathrm{~dB}$.
\end{abstract}

\section{Introduction}

Electrocardiogram (ECG) classification systems require automated measurement and processing capabilities. The first stage in the overall computerized process is beat detection and as such the accuracy of this stage is very important for the overall system performance. The process of beat detection involves the detection of each cardiac cycle in addition to locating the position and the boundaries of each individual waveform component. The most commonly used beat detection approaches are based on non-syntactic methods [1]-[3] and cross-correlation (CC) based algorithms [1]-[2], [4][5]. More recently, artificial intelligence based techniques, using for example neural networks (NN) [2], [6]-[7] have also been used as alternative approaches to ECG beat detection. All of these methods have proposed successful approaches to beat detection. Nevertheless, ongoing research continuously shows the necessity to further improve this process [2], [8]-[11].

One of the major challenges in beat detection is in dealing with noise components present which have the potential to corrupt the underlying signal. Friesen et al. [12] have previously presented details relating to the limitations ECG beat detectors have with respect to ECG signals influenced by noise sources. Ongoing research [10]-[11] demonstrates that researchers are still trying to find and apply new developed techniques, for example empirical mode decomposition (EMD), to provide a more robust method for beat detection.

Within this paper we discuss the effects of three different noise sources on the performance of a number of ECG beat detection algorithms. Motion artifacts (MA noise), EMG interference (EM noise), and possible baseline drifts (BW noise) are three of the most common sources of noise present in ECG recordings. Examples of these different noise sources are shown in Figure 1.

a)
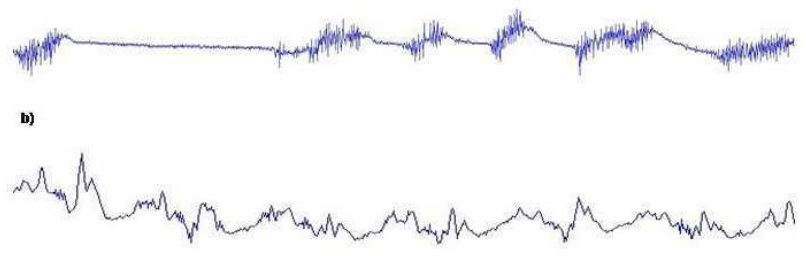

c)

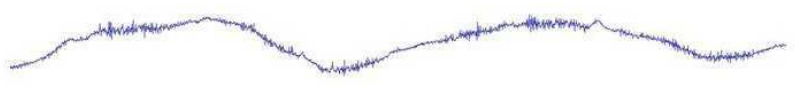

Fig. 1. Example of 3 different noise sources: a) Motion Artifact (MA), b) EMG Interference (EM) and c) Baseline Wander (BW).

MA is caused by changes in the electrode-skin impedance and is often a result of vibrations and movements of the subject. The most important characteristics are the duration (100-500 ms) and its peak-to-peak amplitude which can be up to 500 percent of the ECG signal's peak-to-peak amplitude. Noise signals caused by muscle contractions (EM) can be 
assumed to be transient bursts of zero-mean band-limited Gaussian noise which are usually in the micro volt range. The main characteristics are a standard deviation of about 10 percent of the peak-to-peak ECG amplitude and duration of about $50 \mathrm{~ms}$. The frequency content of the baseline EMG varies from $\mathrm{DC}$ to $10 \mathrm{kHz}$. BW can be seen as a sinusoidal component at the frequency of respiration which is added to the ECG signal. The characteristics of such a baseline drift are the amplitude which varies up to 15 percent of the original ECG signal's peak-to-peak amplitude, and the baseline variation of about 15 percent of this peak-to-peak ECG amplitude at about $0.15 \mathrm{~Hz}$ to $0.3 \mathrm{~Hz}$.

The following section provides an introduction to the four methods of beat detection examined within this study; a non-syntactic approach, a CC based method and two newly developed multi-component based techniques [5]-[6] utilizing CC and NNs. The results section presents a summary of the performance of the beat detectors with respect to their performance in processing ECG signals corrupted by noise sources. Finally, the influences of the noise sources with varying degrees of SNR in addition to the advantages and disadvantages of the four beat detectors are discussed in the Discussion and Conclusion section.

\section{Methods}

\section{A. Non-Syntactic Approach (SRB)}

The non-syntactic technique is one of the most commonly applied and established methods of ECG beat detection [1]-[3]. Such approaches are normally separated into stages of QRS-complex detection and further, gradient based, search algorithms for locating the P-wave and T-wave. The non-syntactic algorithm used within this study is based on the well known approach presented by Pan and Tompkins [3]. Detailed information regarding the algorithm and parameters used for the SRB method are presented in [5].

\section{B. Single-Component based CC Method (SCC)}

The SCC approach is based on the fundamentals of $\mathrm{CC}$, also a commonly used technique [1]-[2], [4]. A PQRST template, which is generated during an initial training phase, is subsequently cross-correlated with a patient's ECG signal to measure the similarities between the two signals. Further details about the SCC method used within this study can be found in [5].

\section{Multi-Component based CC Approach (MCC)}

The fundamental concepts of $\mathrm{CC}$, as used for the previous SCC method, are also used for the $\mathrm{MCC}$ approach. This method extends the SCC approach through its multi-component [5]-[6] based beat detection concept. A multi-component beat detector consists of separate and individual detectors for the individual waveform components (P-wave, QRS-complex, and Twave), each of which perform a parallel analysis of the patient's ECG signal. Such a detector is capable of locating a waveform component without any information about the position of the other components. Further information, describing the multi-component based beat detection technique and in particular the details about the MCC method, can be found in [5].

\section{Multi-Component based NN Approach (MNN)}

The MNN approach can also be considered as a multicomponent based technique [5]-[6]. Three independent detectors are used for P-wave, QRS-complex and T-wave detection. Each detector consists of a multi-layer feed forward NN. The three networks are trained with independent data and are tuned for detection of different waveform components. Detailed information about the MNN approach is presented in [6].

\section{E. Preparation of Noise Test ECG Dataset}

Prior to evaluation of the four different beat detection approaches, three of the most common sources of noise (MA, EM, BW), which may influence ECG recordings, were added to the original signals from the QT database [13]. An example, illustrating this procedure based on one ECG record and MA as the noise source, added to the signal, is presented in Figure 2.

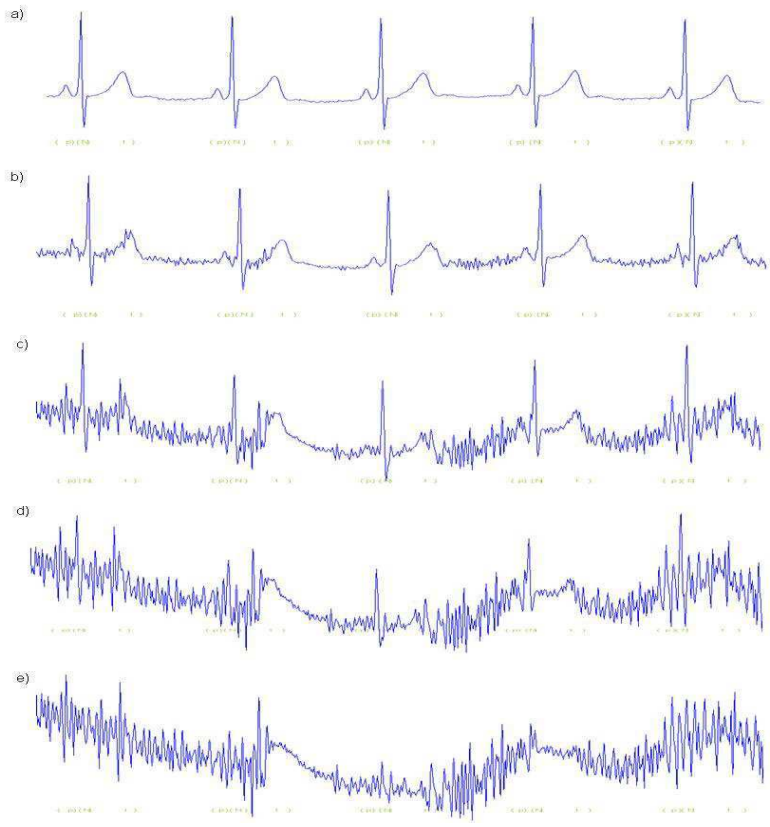

Fig. 2. a) ECG record sel16272 influenced by motion artifact (MA) noise with b) $24 \mathrm{~dB}$ SNR, c) $12 \mathrm{~dB}$ SNR, d) $6 \mathrm{~dB}$ SNR, and e) $-6 \mathrm{~dB}$ SNR. 
The QT database is a collection of ECG recordings, representing a large variety of normal and abnormal signals, from existing databases, e.g. the MIT BIH Arrhythmia database and the European Society of Cardiology ST-T database. A data set of approximately 3000 annotated beats from 100 different ECG records was used to validate the performance of the four beat detection algorithms with regard to noise. The three types of noise were added to the 100 original records at four different SNRs of $24 \mathrm{~dB}, 12 \mathrm{~dB}, 6 \mathrm{~dB}$, and $-6 \mathrm{~dB}$. Hence, 1200 additional records to the 100 clean records were used for testing the performance based on the influence of noise. The three noise sources of MA, EM and BW were provided with the MIT-BIH Noise Stress Test Database [14]. The sources are provided as signal files stored in the same format, the WFDB format, as the ECG records from the QT database. A number of tools, for instance ' $n s t$ ', have been developed from PhysioNet [15], which can be used to add two different signals (e.g. noise signal and ECG record) with defined SNRs.

\section{Results}

In order to validate the four beat detection algorithms using the 1200 ECG records influenced by noise, each detector was trained based on a patient specific training technique [16] using one beat of each of the 100 original ECG records before any form of noise was added. As recommended by the American Standard ANSI/AAMI EC57 [17], the number of correctly detected waveform components (sensitivity), corresponding to the number of true positives (TPs), and the probability of incorrectly detected waveform components (1-specificity), corresponding to the number of false positives (FPs), were measured.

$$
\begin{aligned}
& \text { Sensitivity }=T P S=\frac{100 \% \times N_{c d}}{N_{p}} \\
& 1-\text { Specificity }=F P S=\frac{100 \% \times N_{f d}}{N_{f d}+N_{c d}}
\end{aligned}
$$

These measures are presented in Equation 3.1 and Equation 3.2 where $\mathrm{N}_{\mathrm{cd}}$ is the number of correctly detected markers, $\mathrm{N}_{\mathrm{p}}$ the number of markers present (reference markers) and $\mathrm{N}_{\mathrm{fd}}$ the number of markers detected at incorrect positions.

As an example, Figures 3-5 show a part of the results following comprehensive tests. The QRS-complex detection results of the four beat detection methods (SRB, SCC, MCC and MNN) based on the influence of MA noise with SNRs varying from $24 \mathrm{~dB}$ down to $-6 \mathrm{~dB}$ are summarized in Figure 3. The QRS-complex detection results based on the influence of EM and BW noise is shown in Figure 4 and Figure 5 respectively.

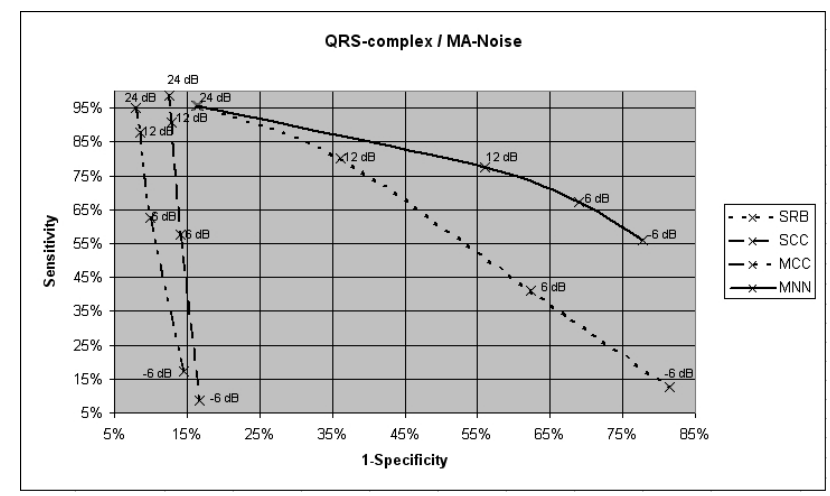

Fig. 3. QRS-complex detection results of methods SRB, SCC, MCC and MNN based on ECG signals influenced by MA noise with SNRs equal to $24 \mathrm{~dB}, 12 \mathrm{~dB}, 6 \mathrm{~dB}$ and $-6 \mathrm{~dB}$.

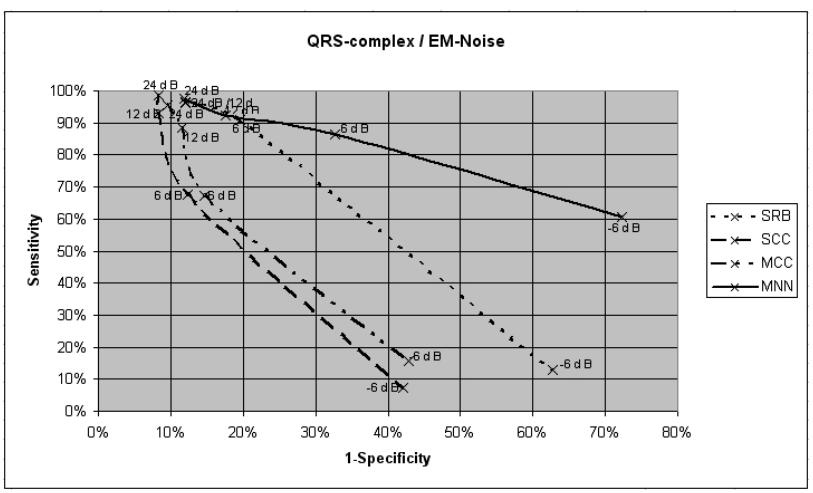

Fig. 4. QRS-complex detection results of methods SRB, SCC, MCC and MNN based on ECG signals influenced by EM noise with SNRs equal to $24 \mathrm{~dB}, 12 \mathrm{~dB}, 6 \mathrm{~dB}$ and $-6 \mathrm{~dB}$.

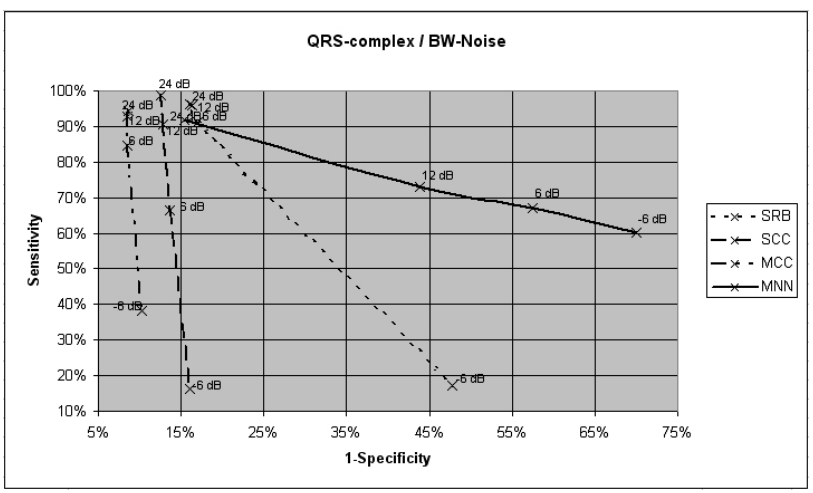

Fig. 5. QRS-complex detection results of methods SRB, SCC, MCC and MNN based on ECG signals influenced by BW noise with SNRs equal to $24 \mathrm{~dB}, 12 \mathrm{~dB}, 6 \mathrm{~dB}$ and $-6 \mathrm{~dB}$.

The curves, representing the results of the four individual methods in detecting QRS-complexes show one common trend for both $\mathrm{CC}$ methods. The sensitivity of the two $\mathrm{CC}$ based methods decreases quickly with SNRs below $12 \mathrm{~dB}$. The results for 1-specificity only increase slightly for SNRs from $24 \mathrm{~dB}$ down to $6 \mathrm{~dB}$. At a SNR of $12 \mathrm{~dB}$, both $\mathrm{CC}$ methods can still detect more 
than $85 \%$ of the QRS-complexes correctly. The SRB method shows a low sensitivity to EM and BW noise for SNRs down to $6 \mathrm{~dB}$. The MNN method outperforms the other three methods in detecting QRS-complexes at low SNRs around $-6 \mathrm{~dB}$. Nevertheless, the probability of detecting QRS-complexes at incorrect positions increases faster for the MNN approach.

The results achieved by the $\mathrm{P}$-wave detectors and $\mathrm{T}$ wave detectors show that in average, the SRB and SCC methods achieved better results than the two multicomponent based approaches, especially at lower SNRs. In most cases, the SCC method outperformed the other three methods for SNRs down to $6 \mathrm{~dB}$.

\section{Discussion and conclusions}

In summary, the results have shown different advantages and disadvantages of the individual detectors depending on the kind of noise and the SNR. Generally both CC based approaches exhibited superior results for SNRs higher than $6 \mathrm{~dB}$. Nevertheless the $\mathrm{CC}$ techniques did show a lower decrease in specificity when increasing the SNR in comparison to the non-syntactic method and the NN based multi-component method. However, the MNN method achieved the highest sensitivity results at low SNRs of $-6 \mathrm{~dB}$.

$\mathrm{CC}$ based techniques are likely better at higher SNRs, since the difference (corresponding to the noise contents) between the actual signal and the template is relatively low for high SNRs. CC functions measure the similarities between a template and the ECG signal and decisions are made based on the comparison of the resulting difference and a threshold. Hence, if the threshold is not too high and the noise contents low, waveform components can still be detected with a high sensitivity. The performance of $\mathrm{NN}$ based methods with respect to noise strongly depends on the early stages such as training of the networks. The noise sensitivity of such methods might be reduced when considering possible noise sources during training. Nevertheless, this would require the knowledge that certain noise sources are present in the ECG.

\section{References}

[1] Nugent CD, Webb JAC, Wright GTH, Black ND. Electrocardiogram I: Pre-processing prior to classification. Automedica, 1998, 16:263-288.

[2] Köhler BU, Henning C, Orglmeister R. The principles of Software QRS Detection: Reviewing and Comparing Algorithms for Detecting this Important ECG Waveform. IEEE Engineering in Medicine and Biology, vol. 2, pp.4257, January/February 2001.

[3] Pan J, Tompkins WJ. A real-time QRS detection algorithm. IEEE Transactions on Biomedical Engineering, 1985, 32:230-236.

[4] Abboud S, Sadeh D. The use of cross-correlation function for the alignment of ECG waveforms and rejection of extrasystoles. Computers and Biomedical Research, 1984, 17:258-266.

[5] Last T, Nugent CD, Owens FJ. Multi-component based cross correlation beat detection in electrocardiogram analysis. BioMedical Engineering OnLine, 2004, 3:26.

[6] Last T, Nugent CD, Owens FJ. Multi-Component Based Neural Network Beat Detection in Electrocardiogram Analysis. Computers in Cardiology, 2006, 33:573-576.

[7] Xue Q, Hu YH, Tompkins WJ., Neural-network based adaptive matched filtering for QRS detection. Med. Biol. Eng. Conf., Montreal, Quebec, Canada, 1997, pp.10511052.

[8] Zhang Q, Manriquez I, Mdeique A, Papelier C, Sorine Y. An Algorithm for Robust and Efficient Location of TWave Ends in Electrocardiograms. IEEE Transactions on Biomedical Engineering, vol. 53, issue 12, pp.2544-2552, 2006.

[9] Matinez JP, Almeida R, Olmos S, Laguna P. A waveletbased ECG delineator: evaluation on standard databases. IEEE Transactions on Biomedical Engineering, vol. 51, 4:570-581, 2004.

[10] Weng B, Blanco-Velasco M, Barner KE. Baseline Wander Correction in ECG by the Empirical Mode Decomposition. IEEE Bioengineering Conference 2006, pp.135-136, 2006.

[11] Amit J, Nimunkar, Tompkins WJ. R-peak Detection and Signal Averaging for Simulated Stress ECG using EMD. IEEE International Conference of Engineering in Medicine and Biology Society, 2007, pp.1261-1264.

[12] Friesen GM, Jannett TC, Jadallah MA, Yates SL, Quint SR, Nagle HT. A comparison of the noise sensitivity of nine QRS detection algorithms. IEEE Transactions on Biomedical Engineering, vol.37, no.1, pp. 85-98, 1990.

[13] Laguna P, Mark RG, Goldberger A, Moody DG. A database for evaluation of algorithms for measurement the QT and other waveform intervals in the ECH. Computers in Cardiology, 1997, 24:673-676.

[14] Massachusetts Institute of Technology. MIT-BIH ECG database. http://ecg.mit.edu/.

[15] Massachusetts Institute of Technology. PhysioNet Resource Center. http://www.physionet.org/.

[16] Last T, Nugent CD, Owens FJ. Evaluation of electrocardiogram beat detection algorithms: patient specific versus generic training. International Conference of the IEEE Engineering in Medicine and Biology Society, 2007, pp.3208-3211.

[17] ANSI/AAMI EC57: Testing and reporting performance results of cardiac rhythm and ST segment measurement algorithms (AAMI Recommended Practice / American National Standard), 1998, Available: http://www.aami.org.

Address for correspondence:

Thorsten Last,

Hussenstrasse 31, 78462 Konstanz, Germany

E-mail: tote_last@yahoo.de 